Bull. Korean Math. Soc. 52 (2015), No. 1, pp. 85-103

http://dx.doi.org/10.4134/BKMS.2015.52.1.085

\title{
ON DISTANCE ESTIMATES AND ATOMIC DECOMPOSITIONS IN SPACES OF ANALYTIC FUNCTIONS ON STRICTLY PSEUDOCONVEX DOMAINS
}

\author{
Miloš Arsenović And Romi F. Shamoyan
}

\begin{abstract}
We prove some sharp extremal distance results for functions in various spaces of analytic functions on bounded strictly pseudoconvex domains with smooth boundary. Also, we obtain atomic decompositions in multifunctional Bloch and weighted Bergman spaces of analytic functions on strictly pseudoconvex domains with smooth boundary, which extend known results in the classical case of a single function.
\end{abstract}

\section{Introduction}

Throughout this paper $H(D)$ denotes the space of all holomorphic functions on an open set $D \subset \mathbb{C}^{n}$. We follow notation from [14]. Let $D$ be a bounded strictly pseudoconvex domain in $\mathbb{C}^{n}$ with smooth boundary, let $d(z)=\operatorname{dist}(z, \partial D)$. Then there is a neighborhood $U$ of $\bar{D}$ and $\rho \in C^{\infty}(U)$ such that $D=\{z \in U: \rho(z)>0\},|\nabla \rho(z)| \geq c>0$ for $z \in \partial D, 0<\rho(z)<1$ for $z \in D$ and $-\rho$ is strictly plurisubharmonic in a neighborhood $U_{0}$ of $\partial D$. Note that $d(z) \asymp \rho(z), z \in D$. Then there is an $r_{0}>0$ such that the domains $D_{r}=\{z \in D: \rho(z)>r\}$ are also smoothly bounded strictly pseudoconvex domains for all $0 \leq r \leq r_{0}$. Let $d \sigma_{r}$ be the normalized surface measure on $\partial D_{r}$ and $d V$ the Lebesgue measure on $\mathbb{C}^{n}$. The following mixed norm spaces were investigated in [14]. For $0<p<\infty, 0<q \leq \infty, \delta>0$ and $k=0,1,2, \ldots$ set

$$
\|f\|_{p, q, \delta ; k}=\left(\sum_{|\alpha| \leq k} \int_{0}^{r_{0}}\left(r^{\delta} \int_{\partial D_{r}}\left|D^{\alpha} f\right|^{p} d \sigma_{r}\right)^{q / p} \frac{d r}{r}\right)^{1 / q}, \quad 0<q<\infty
$$

and

$$
\|f\|_{p, \infty, \delta ; k}=\sup _{0<r<r_{0}} \sum_{|\alpha| \leq k}\left(r^{\delta} \int_{\partial D_{r}}\left|D^{\alpha} f\right|^{p} d \sigma_{r}\right)^{1 / p} .
$$

Received April 15, 2013; Revised July 31, 2013.

2010 Mathematics Subject Classification. Primary 30D45.

Key words and phrases. Bergman spaces, Bloch space, pseudoconvex domains, extremal problems, atomic decomposition.

The first author was supported by Ministry of Science, Serbia, project OI174017. 
The corresponding spaces $A_{\delta ; k}^{p, q}=A_{\delta ; k}^{p, q}(D)=\left\{f \in H(D):\|f\|_{p, q, \delta ; k}<\infty\right\}$ are complete quasi normed spaces, for $p, q \geq 1$ they are Banach spaces. We mostly deal with the case $k=0$, then we write simply $A_{\delta}^{p, q}$ and $\|f\|_{p, q, \delta}$. We also consider these spaces for $p=\infty$ and $k=0$, the corresponding space is denoted by $A_{\delta}^{\infty, p}=A_{\delta}^{\infty, p}(D)$ and consists of all $f \in H(D)$ such that

$$
\|f\|_{\infty, p, \delta}=\left(\int_{0}^{r_{0}}\left(\sup _{\partial D_{r}}|f|\right)^{p} r^{\delta p-1} d r\right)^{1 / p}<\infty .
$$

Also, for $\delta>-1$, the space $A_{\delta}^{\infty}=A_{\delta}^{\infty}(D)$ consist of all $f \in H(D)$ such that

$$
\|f\|_{A_{\delta}^{\infty}}=\sup _{z \in D}|f(z)| \rho(z)^{\delta}<\infty,
$$

and the weighted Bergman space $A_{\delta}^{p}=A_{\delta}^{p}(D)=A_{\delta+1}^{p, p}(D)$ consists of all $f \in$ $H(D)$ such that

$$
\|f\|_{A_{\delta}^{p}}=\left(\int_{D}|f(z)|^{p} \rho^{\delta}(z) d V(z)\right)^{1 / p}<\infty .
$$

The space of all $f \in H(D)$ such that $\sup _{z \in D}|D f(z)| \rho(z)=\|f\|_{\mathcal{B}}<+\infty$ is called Bloch space on $D$ and denoted by $\mathcal{B}$. Here $D f=\left(\partial f / \partial z_{1}, \ldots, \partial f / \partial z_{n}\right)$. This space is a Banach space, if one identifies functions which differ by a constant.

The next section is a continuation of our previous work [18] and treats the following problem: estimate $\operatorname{dist}_{Y}(f, X), f \in Y$ where $(X, Y)$ is one of the following pairs: $\left(A_{\delta}^{1, p}, A_{\delta}^{1, \infty}\right)$ and $\left(A_{\delta}^{\infty, p}, A_{\delta}^{\infty}\right)$. In both cases we give sharp results. Techniques used to obtain our results were previously used to study analogous problems for analytic Besov spaces in the unit ball and polydisc (see [19], [20]). The literature on the extremal problems in spaces of analytic functions is extensive, even in the case of the unit disk, a classical exposition of these problems treated by duality methods developed by S. Havinson, W. Rogosinski and H. Shapiro can be found in [7].

Now we recall some of the definitions and results from [14].

Definition 1. For $\epsilon>0, \nu \in \mathbb{R}, 0<p<\infty$ and $0<q \leq \infty$ mixed norm double sequence spaces $l_{\nu}^{p, q, \epsilon}$ are defined by: $c=\left(c_{m, j}\right)_{m, j=0}^{\infty} \in l_{\nu}^{p, q, \epsilon}$ if

$$
\|c\|_{p, q, \nu, \epsilon}=\left(\sum_{m=0}^{\infty}\left(\sum_{j=0}^{\infty}\left|c_{m, j}\right|^{p}\right)^{q / p} \epsilon^{m \nu q / p}\right)^{1 / q}
$$

with the usual modification if $q=\infty$.

Clearly this definition applies also in the case where the range of indices is $m \geq 0,0 \leq j \leq j_{m}$. Note that these spaces depend on 3 parameters, namely $p, q$ and $\epsilon^{\nu}$. 
Lemma 1 ([14]). Let $d_{K}$ be Koranyi pseudodistance in $D$, let $B_{K}(\zeta, \epsilon)$ be the Koranyi ball centered at $\zeta$ of radius $\epsilon>0$. Then for each $\eta>0$ and $0<\epsilon<1$ there exist $0<\eta_{0}<\eta_{1}<\eta$ and a sequence $\left\{a_{m, j}\right\}, m=0,1, \ldots, j=0, \ldots, j_{m}$ of points in $D$ such that:

$$
\begin{aligned}
& \cup_{m, j} B_{K}\left(a_{m, j}, \eta_{1} d\left(a_{m, j}\right)\right)=D, \\
& B_{K}\left(a_{m, j}, \eta_{0} d\left(a_{m, j}\right)\right) \cap B_{K}\left(a_{m^{\prime}, j^{\prime}}, \eta_{0} d\left(a_{m^{\prime}, j^{\prime}}\right)\right) \neq \emptyset \quad \text { if and only if } \\
& m=m^{\prime}, j=j^{\prime}, \rho\left(a_{m, j}\right) \asymp \epsilon^{m} .
\end{aligned}
$$

Let us call such a system of points in $D$ an $\eta_{0}-\eta_{1}$ lattice in $D$. These lattices play an important role in investigation of spaces of analytic functions on $D$ and operators acting on them. As an example of such applications in the case of the unit ball in $\mathbb{C}^{n}$ see [17]; we note that these results on Lusin's area operator acting on weighted Bergman spaces can be extended to the present context using recent results from [1].

Theorem 1 ([14]). Let $\left\{a_{m, j}\right\}$ be an $\eta_{0}-\eta_{1}$ lattice in D, as in Lemma 1. Let us set, for a double sequence $c=\left\{c_{m, j}\right\}, m \geq 0,0 \leq j \leq j_{m}$ :

$$
T_{s}(c)=\sum_{m=0}^{\infty} \sum_{j=0}^{j_{m}} c_{m, j} K^{n+1+s, s}\left(a_{m, j}, z\right), \quad z \in D .
$$

For $s>\delta / p$ this operator is continuous and onto from $l_{n+\delta-k p}^{p, q, \epsilon}$ to $A_{\delta, k}^{p, q}(D)$.

We refer reader to [14] for definition of kernels $K^{n+1+s, s}(\zeta, z)$.

Clearly, the above theorem is a result on atomic decomposition of the spaces $A_{\delta, k}^{p, q}(D)$. It is a natural question to generalize this result to the multifunctional setting.

We define, for $m \in \mathbb{N}, 0<p_{1}, \ldots, p_{m}<\infty, \alpha_{1}, \ldots, \alpha_{m}>-1$ the weighted multifunctional Bergman space $M A_{\tilde{p}, \tilde{\alpha}}(D)=M A_{\tilde{p}, \tilde{\alpha}}, \tilde{p}=\left(p_{1}, \ldots, p_{m}\right), \tilde{\alpha}=$ $\left(\alpha_{1}, \ldots, \alpha_{m}\right)$ as the space of all $m$-tuples $\left(f_{1}, \ldots, f_{m}\right)$ of holomorphic functions in $D$ such that

$$
\left(f_{1}, \ldots, f_{m}\right)_{\tilde{p}, \tilde{\alpha}}=\int_{D} \prod_{j=1}^{m}\left|f_{j}(z)\right|^{p_{j}} \rho(z)^{(m-1)(n+1)+\sum_{j=1}^{m} \alpha_{j}} d V(z)<\infty .
$$

In the third section we generalize Theorem 1, in the special case of weighted Bergman spaces, to the case of multifunction spaces over strictly pseudoconvex domains. For the case of the unit ball in $\mathbb{C}^{n}$, see [11]. Moreover, we prove an atomic decomposition theorem for multifunctional Bloch space $M B(m)$ consisting of all $m$-tuples $\left(f_{1}, \ldots, f_{m}\right)$ of functions analytic in $D$ such that

$$
\left(f_{1}, \ldots, f_{m}\right)_{M B(m)}=\sup _{z \in D}\left|\nabla\left(f_{1} \cdots f_{m}\right)(z)\right| d(z) \log ^{1-m} \frac{1}{d(z)}<\infty,
$$

generalizing a classical result $(m=1)$ on atomic decomposition of Bloch functions, see [5]. 
We use common convention regarding constants: letter $C$ denotes a constant which can change its value from one occurrence to the next one.

\section{Distance estimates in $A_{\delta}^{p, q}(D)$ and $A_{\delta}^{\infty}(D)$ spaces}

Our proofs are heavily based on the estimates from [3], where more general situation was considered.

Since $|f(z)|^{p}$ is subharmonic (even plurisubharmonic) for a holomorphic $f$, we have $A_{s}^{p}(D) \subset A_{t}^{\infty}(D)$ for $0<p<\infty$, sp $>n$ and $t=s$. Also, $A_{s}^{p}(D) \subset$ $A_{s}^{1}(D)$ for $0<p \leq 1$ and $A_{s}^{p}(D) \subset A_{t}^{1}(D)$ for $p>1$ and $t$ sufficiently large. Therefore we have an integral representation

$$
f(z)=\int_{D} f(\xi) K(z, \xi) \rho^{t}(\xi) d V(\xi), \quad f \in A_{t}^{1}(D), \quad z \in D
$$

where $K(z, \xi)$ is a kernel of type $t$, that is a smooth function on $D \times D$ such that $|K(z, \xi)| \leq C|\tilde{\Phi}(z, \xi)|^{-(n+1+t)}$, where $\tilde{\Phi}(z, \xi)$ is so called Henkin-Ramirez function for $D$. Note that (1) holds for functions in any space $X$ that embeds into $A_{t}^{1}$. We review some facts on $\tilde{\Phi}$ and refer reader to [16] for details. This function is $C^{\infty}$ in $U \times U$, where $U$ is a neighborhood of $\bar{D}$, it is holomorphic in $z$, and $\tilde{\Phi}(\zeta, \zeta)=\rho(\zeta)$ for $\zeta \in U$. Moreover, on $\bar{D} \times \bar{D}$ it vanishes only on the diagonal $(\zeta, \zeta), \zeta \in \partial D$. Locally, it is up to a non vanishing smooth multiplicative factor equal to the Levi polynomial of $\rho$. From now on we work with a fixed Henkin-Ramirez function $\tilde{\Phi}$. We are going to use the following results from [3] and [2].

Lemma 2. Assume $K(z, \xi)$ is a kernel of type $t, t>-1$.

a) For $0<r<r_{0}$ we have

$$
\int_{\partial D_{r}}|K(z, \zeta)| d \sigma_{r}(z) \leq C(\rho(\zeta)+r)^{-t-1}, \quad \zeta \in D .
$$

b) Assume $\sigma>0$ satisfies $\sigma-t-1<0$. Then we have

$$
\int_{D}|K(z, \zeta)| \rho^{\sigma-1}(z) d V(z) \leq C \rho^{\sigma-t-1}(\zeta) \quad \zeta \in D .
$$

For part a) of the above lemma see Corollary 3.9. of [3], for part b) see [2]. We note that the same estimates are valid if $K$ is replaced by $\tilde{K}(z, \zeta)=K(\zeta, z)$.

The estimates of such type for strictly pseudoconvex domains have a long history, the basis for such results were constructive methods in several complex variables, namely integral representation formulas developed by Henkin and Ramirez around 1970. E. Ligocka obtained an important factorization theorem for the weighted Bergman kernel, see [13], building on the previous work by Kerzman and Stein, see [8]. Then, using results from [13], P. Ahern and R. Schneider obtained estimate contained in Lemma 2, which is of vital importance for our work. For further results in this directions see [2], [4] and recent papers [1], [6]. 
Each of the embedding results we cite below poses the corresponding problem of distance estimates, and in each of these cases we provide at least a partial answer. In most cases we provide two-sided estimates.

Proposition 1 ([14]). If $0<p_{0}<p_{1}<\infty, 0<q \leq \infty, \delta_{0}, \delta_{0}^{\prime}>0$ and $\frac{n+\delta_{0}^{\prime}}{p_{1}}=\frac{n+\delta_{0}}{p_{0}}$, then $A_{\delta_{0} ; k}^{p_{0}, q}(D) \hookrightarrow A_{\delta_{0}^{\prime} ; k}^{p_{1}, q}(D)$.

As a special case of the above proposition we have the following result.

Lemma 3. If $0<p \leq 1$ and $\alpha>-1$, then

$$
A_{\alpha}^{p}(D) \hookrightarrow A_{\beta}^{1}(D), \quad \beta=\frac{n+1+\alpha}{p}-(n+1) .
$$

Equivalently:

$$
\int_{D}|F(z)| \rho(z)^{\frac{n+1+\alpha}{p}-(n+1)} d V(z) \leq C\|F\|_{A_{\alpha}^{p}}, \quad F \in H(D) .
$$

Proposition 2 ([14]). If $0<p<\infty, 0<q_{0}<q_{1} \leq \infty, \delta>0$ and $k=$ $0,1,2, \ldots$, then $A_{\delta ; k}^{p, q_{0}}(D) \hookrightarrow A_{\delta ; k}^{p, q_{1}}(D)$.

In particular, $A_{\delta}^{1, p} \hookrightarrow A_{\delta}^{1, \infty}$. This result motivates the following problem: Estimate the distance from a given function $f \in A_{\delta}^{1, \infty}$ to $A_{\delta}^{1, p}$. The solution to this problem is given in the following two theorems. We set, for $\epsilon>0$ and $\delta>0$ :

$$
L_{\epsilon, \delta}(f)=\left\{r \in\left(0, r_{0}\right): r^{\delta} \int_{\partial D_{r}}|f| d \sigma_{r} \geq \epsilon\right\} .
$$

Theorem 2. Let $1 \leq p<\infty, \delta>0$ and $f \in A_{\delta}^{1, \infty}(D)$. Set

$$
\begin{gathered}
s_{1}(f)=\operatorname{dist}_{A_{\delta}^{1, \infty}}\left(f, A_{\delta}^{1, p}\right), \\
s_{2}(f)=\inf \left\{\epsilon>0: \int_{0}^{1} \chi_{L_{\epsilon, \delta}(f)}(r) \frac{d r}{r}<\infty\right\} .
\end{gathered}
$$

Then $s_{1}(f) \asymp s_{2}(f)$.

Proof. Assume $s_{2}(f)<s_{1}(f)$. Then there are $\epsilon>\epsilon_{1}>0$ and $f_{1} \in A_{\delta}^{1, p}$ such that $\left\|f-f_{1}\right\|_{1, p, \delta} \leq \epsilon_{1}$ and

$$
\int_{0}^{r_{0}} \chi_{L_{\epsilon, \delta}(f)}(r) \frac{d r}{r}=+\infty .
$$

Therefore we have

$$
\begin{aligned}
r^{\delta} \int_{\partial D_{r}}\left|f_{1}\right| d \sigma_{r} & \geq r^{\delta} \int_{\partial D_{r}}|f| d \sigma_{r}-\sup _{0<R<r_{0}} R^{\delta} \int_{\partial D_{R}}\left|f-f_{1}\right| d \sigma_{R} \\
& \geq r^{\delta} \int_{\partial D_{r}}|f| d \sigma_{r}-\epsilon_{1}
\end{aligned}
$$


for every $0<r<r_{0}$. Hence the following estimate from below

$$
r^{\delta} \int_{\partial D_{r}}\left|f_{1}\right| d \sigma_{r} \geq r^{\delta} \int_{\partial D_{r}}|f| d \sigma_{r}-\epsilon_{1} \geq \epsilon-\epsilon_{1} .
$$

holds for every $r \in L_{\epsilon, \delta}(f)$. However, this implies:

$$
\begin{aligned}
\left(\epsilon-\epsilon_{1}\right)^{p} \int_{0}^{r_{0}} \chi_{L_{\epsilon, \delta}(f)}(r) \frac{d r}{r} & \leq \int_{0}^{r_{0}}\left(r^{\delta} \int_{\partial D_{r}}\left|f_{1}\right| d \sigma_{r}\right)^{p} \frac{d r}{r} \\
& =\|f\|_{1, p, \delta}^{p}<\infty,
\end{aligned}
$$

which contradicts (4). Hence we proved $s_{1}(f) \leq s_{2}(f)$.

Next we prove $s_{2}(f) \leq C s_{1}(f)$. Let us choose $f \in A_{\delta}^{1, \infty}$ and fix an $\epsilon>0$ such that the integral appearing in (3) is finite. Since $A_{\delta}^{1, \infty} \hookrightarrow A_{\delta}^{1}$, we can use integral representation (1) for function $f$ :

$$
\begin{aligned}
f(z)= & \int_{D} f(\zeta) K(z, \zeta) \rho^{t}(\zeta) \chi_{L_{\epsilon, \delta}(f)}(\rho(\zeta)) d V(\zeta) \\
& +\int_{D} f(\zeta) K(z, \zeta) \rho^{t}(\zeta)\left[1-\chi_{L_{\epsilon, \delta}(f)}(\rho(\zeta))\right] d V(\zeta) \\
= & f_{1}(z)+f_{2}(z)
\end{aligned}
$$

The proof will be completed once we show the following two estimates:

$$
\begin{gathered}
\left\|f_{1}\right\|_{1, p, \delta} \leq C\|f\|_{1, \infty, \delta}, \\
\left\|f_{2}\right\|_{1, \infty, \delta} \leq C \epsilon .
\end{gathered}
$$

Since, by Proposition 2, we have $\left\|f_{1}\right\|_{1, p, \delta} \leq C\left\|f_{1}\right\|_{1,1, \delta}$ it suffices, in proving estimate (6), to assume $p=1$. Set $L=L_{\epsilon, \delta}(f)$. We have, using Lemma 2 and Fubini's theorem:

$$
\begin{aligned}
\left\|f_{1}\right\|_{1,1, \delta} & =\int_{0}^{r_{0}}\left(\int_{\partial D_{r}}\left|f_{1}(z)\right| d \sigma_{r}(z)\right) r^{\delta-1} d r \\
& \leq \int_{0}^{r_{0}}\left(\int_{\partial D_{r}}\left(\int_{D}\left|f(\zeta) K(z, \zeta) \rho^{t}(\zeta) \chi_{L}(\rho(\zeta))\right| d V(\zeta)\right) d \sigma_{r}(z)\right) r^{\delta-1} d r \\
& =\int_{D}|f(\zeta)| \rho^{t}(\zeta) \chi_{L}(\rho(\zeta)) \int_{0}^{r_{0}} \int_{\partial D_{r}}|K(z, \zeta)| d \sigma_{r}(z) r^{\delta-1} d r d V(\zeta) \\
& \leq C \int_{D}|f(\zeta)| \rho^{t}(\zeta) \chi_{L}(\rho(\zeta)) \int_{D}|K(z, \zeta)| \rho^{\delta-1} d V(z) d V(\zeta) \\
& \leq C \int_{D}|f(\zeta)| \rho^{\delta}(\zeta) \chi_{L}(\rho(\zeta)) d V(\zeta) \\
& \leq C\|f\|_{1, \infty, \delta} \int_{0}^{r_{0}} \chi_{L}(r) \frac{d r}{r} \\
& \leq C_{\epsilon}\|f\|_{1, \infty, \delta} .
\end{aligned}
$$


Next we prove estimate (7). For $0<r<r_{0}$ we have, using Fubini's theorem, Lemma 2 and definition of the set $L=L_{\epsilon, \delta}$ :

$$
\begin{aligned}
& r^{\delta} \int_{\partial D_{r}}\left|f_{2}(z)\right| d \sigma_{r}(z) \\
\leq & r^{\delta} \int_{\partial D_{r}} \int_{D}|f(\zeta) K(z, \zeta)| \rho^{t}(\zeta)\left[1-\chi_{L}(\rho(\zeta))\right] d V(\zeta) d \sigma_{r}(z) \\
= & r^{\delta} \int_{D}|f(\zeta)| \rho^{t}(\zeta)\left[1-\chi_{L}(\rho(\zeta))\right] \int_{\partial D_{r}}|K(z, \zeta)| d \sigma_{r}(z) d V(\zeta) \\
\leq & C r^{\delta} \int_{D}|f(\zeta)| \rho^{t}(\zeta)(r+\rho(\zeta))^{-t-1}\left[1-\chi_{L}(\rho(\zeta))\right] d V(\zeta) \\
\leq & C r^{\delta} \int_{0}^{r_{0}} \int_{\partial D_{R}}|f(\zeta)| \frac{R^{t}}{(r+R)^{t+1}}\left[1-\chi_{L}(R)\right] d \sigma_{R}(\zeta) d R \\
\leq & C \epsilon r^{\delta} \int_{0}^{r_{0}} \frac{R^{t-\delta}}{(r+R)^{t+1}} d R \\
\leq & C \epsilon
\end{aligned}
$$

and the proof is complete.

The next theorem complements the previous one, it deals with the case $0<p \leq 1$.

Theorem 3. Let $0<p \leq 1, \delta>0, t>\delta-1$ and $f \in A_{\delta}^{1, \infty}$. Set

$$
\tilde{s}_{1}(f)=\operatorname{dist}_{A_{\delta}^{1, \infty}}\left(f, A_{\delta}^{1, p}\right)
$$

and

(9) $\tilde{s}_{2}(f)=\inf \left\{\epsilon>0: \int_{0}^{1}\left(\int_{0}^{1} \chi_{L_{\epsilon, \delta}(f)}(r) \frac{r^{t-\delta} d r}{(r+\rho)^{t+1}}\right)^{p} \rho^{p \delta-1} d \rho<\infty\right\}$.

Then $\tilde{s}_{1}(f) \asymp \tilde{s}_{2}(f)$.

Proof. We rely on the method used in [20] to prove an analogous result in the one dimensional setting. Inequality $\tilde{s}_{2}(f) \leq \tilde{s}_{1}(f)$ is proved by a standard argument, as in the previous theorem. Let us prove the estimate $\tilde{s}_{1}(f) \leq$ $C \tilde{s}_{2}(f)$. We choose $f \in A_{\delta}^{1, \infty}$ and $\epsilon>0$ such that the integral in (9) is finite. Set $L=L_{\epsilon, \delta}(f)$ and use the same decomposition $f(z)=f_{1}(z)+f_{2}(z)$ as in the proof of Theorem 2. Again we have to prove estimates (6) and (7).

We fix $0<R<r_{0}$ and obtain, for $z \in \partial D_{R}$ :

$$
\left|f_{2}(z)\right| \leq C \int_{0}^{r_{0}}\left[1-\chi_{L}(r)\right] \int_{\partial D_{r}}|f(\zeta)||K(z, \zeta)| d \sigma_{r} d r .
$$

Integration over $\partial D_{R}$ yields, with help of Lemma 2 and Fubini's theorem:

$$
\int_{\partial D_{R}}\left|f_{2}(z)\right| d \sigma_{R}(z)
$$




$$
\begin{aligned}
& \leq C \int_{0}^{r_{0}}\left[1-\chi_{L}(r)\right] r^{t} \int_{\partial D_{r}}|f(\zeta)| \int_{\partial D_{R}}|K(z, \zeta)| d \sigma_{R}(z) d \sigma_{r} d r \\
& \leq \int_{0}^{r_{0}}\left[1-\chi_{L}(r)\right] r^{t} \int_{\partial D_{r}} \frac{|f(\zeta)|}{(R+r)^{t+1}} d \sigma_{r} d r \\
& \leq \epsilon \int_{0}^{r_{0}} \frac{r^{t-\delta}}{(R+r)^{t+1}} \\
& \leq C \epsilon R^{-\delta}
\end{aligned}
$$

where we also used definition of the set $L=L_{\epsilon, \delta}(f)$. This proves estimate (7). Finally we prove (6), again using Fubini's theorem and Lemma 2:

$$
\begin{aligned}
& \left\|f_{1}\right\|_{1, p, \delta}^{p} \\
= & \int_{0}^{r_{0}} r^{\delta p-1}\left(\int_{\partial D_{r}}\left|f_{1}\right| d \sigma_{r}\right)^{p} d r \\
\leq & C \int_{0}^{r_{0}} r^{\delta p-1}\left(\int_{\partial D_{r}} \int_{D} \chi_{L}(\rho(\zeta)) \rho(\zeta)^{t}|f(\zeta)||K(z, \zeta)| d V(\zeta) d \sigma_{r}\right)^{p} d r \\
\leq & C \int_{0}^{r_{0}}\left(\int_{0}^{r_{0}} \chi_{L}(R) R^{t} \int_{\partial D_{R}}|f(\zeta)| \int_{\partial D_{r}}|K(z, \zeta)| d \sigma_{r}(z) d \sigma_{r}(\zeta) d R\right)^{p} d r \\
\leq & C \int_{0}^{r_{0}} r^{\delta p-1}\left(\int_{0}^{r_{0}} \chi_{L}(R) R^{t}(r+R)^{-t-1} \int_{\partial D_{R}}|f(\zeta)| d \sigma_{R}(\zeta) d R\right)^{p} d r \\
\leq & C\|f\|_{1, \infty, \delta}^{p} \int_{0}^{r_{0}}\left(\int_{0}^{r_{0}} \chi_{L}(R) \frac{R^{t-\delta}}{(R+r)^{t+1}} d R\right)^{r^{\delta p-1}} d r \\
\leq & C_{\epsilon}\|f\|_{1, \infty, \delta}^{p} .
\end{aligned}
$$

Proposition 3. If $0<p<\infty$ and $\delta>0$, then $A_{\delta}^{\infty, p} \hookrightarrow A_{\delta}^{\infty}$.

This is a well known result, a simple proof can be based on monotonicity of $M_{\infty}(f, r)=\sup _{\partial D_{r}}|f|$ on $r \in\left(0, r_{0}\right)$.

Again, in order to formulate the corresponding distance result we need the following notation:

$$
\tilde{L}_{\epsilon, \delta}(f)=\left\{0<r<r_{0}: r^{\delta} \sup _{\partial D_{r}}|f| \geq \epsilon\right\} .
$$

Theorem 4. Let $\delta>0,1 \leq p<\infty$ and $f \in A_{\delta}^{\infty}$. Set

$$
l_{1}(f)=\operatorname{dist}_{A_{\delta}^{\infty}}\left(f, A_{\delta}^{\infty, p}\right)
$$

and

$$
l_{2}(f)=\inf \left\{\epsilon>0: \int_{0}^{r_{0}} \chi_{\tilde{L}_{\epsilon, \delta}(f)}(r) \frac{d r}{r}<\infty\right\} .
$$

Then $l_{1}(f) \asymp l_{2}(f)$. 
Proof. Let us assume $l_{1}(f)<l_{2}(f)$. Then there are $0<\epsilon_{1}<\epsilon$ and $f_{1} \in A_{\delta}^{\infty, p}$ such that $\left\|f-f_{1}\right\|_{A_{\delta}^{\infty}} \leq \epsilon_{1}$ and

$$
\int_{0}^{1} \chi_{\tilde{L}_{\epsilon, \delta}(f)}(r) \frac{d r}{r}=\infty .
$$

Next we obtain, using $\left\|f-f_{1}\right\|_{A_{\delta}^{\infty}} \leq \epsilon_{1}$ the following inequality:

$$
r^{\delta}\left|f_{1}(z)\right|=\left|r^{\delta} f(z)-r^{\delta}\left[f(z)-f_{1}(z)\right]\right| \geq r^{\delta}|f(z)|-\epsilon, \quad z \in \partial D_{r} .
$$

Hence, for $r \in \tilde{L}_{\epsilon, \delta}(f)$ we have

$$
r^{\delta} \sup _{\partial D_{r}}\left|f_{1}\right| \geq r^{\delta} \sup _{\partial D_{r}}|f|-\epsilon_{1} \geq \epsilon-\epsilon_{1},
$$

or, equivalently,

$$
\chi_{\tilde{L}_{\epsilon, \delta}(f)}(r) \leq \frac{r^{\delta} \sup _{\partial D_{r}}\left|f_{1}\right|}{\epsilon-\epsilon_{1}} .
$$

But this combined with (12) leads to a contradiction with $f_{1} \in A_{\delta}^{\infty, p}$ : it suffices to raise both sides of the above inequality to $p$-th power and integrate over $0<r<r_{0}$ with respect to the measure $d r / r$.

Next we prove $l_{2}(f) \leq C l_{1}(f)$. We fix $f \in A_{\delta}^{\infty}$ and choose $\epsilon>0$ such that the integral appearing in (11) is finite. Since $A_{\delta}^{\infty} \hookrightarrow A_{\delta}^{1}$, we can use representation formula (1) as in the proof of Theorem 2 :

$$
\begin{aligned}
f(z)= & \int_{D} f(\zeta) K(z, \zeta) \rho^{t}(\zeta) \chi_{\tilde{L}_{\epsilon, \delta}(f)}(\rho(\zeta)) d V(\zeta) \\
& +\int_{D} f(\zeta) K(z, \zeta) \rho^{t}(\zeta)\left[1-\chi_{\tilde{L}_{\epsilon, \delta}(f)}(\rho(\zeta))\right] d V(\zeta) \\
= & f_{1}(z)+f_{2}(z)
\end{aligned}
$$

Again, it suffices to prove the following two estimates:

$$
\left\|f_{2}\right\|_{A_{\delta}^{\infty}} \leq C \epsilon
$$

and

$$
\left\|f_{1}\right\|_{A_{\delta}^{\infty, p}} \leq C\|f\|_{A_{\delta}^{\infty}} .
$$

Let us choose $z \in \partial D_{r}, 0<r<r_{0}$. Set $\tilde{L}=\tilde{L}_{\epsilon, \delta}(f)$. Then we have, using Fubini's theorem and Lemma 2:

$$
\begin{aligned}
\left|f_{2}(z)\right| & \leq \int_{D}|f(\zeta) K(z, \zeta)| \rho^{t}(\zeta)\left[1-\chi_{\tilde{L}}(\rho(\zeta))\right] d V(\zeta) \\
& \leq C \int_{0}^{r_{0}} \int_{\partial D_{R}}|f(\zeta) K(z, \zeta)| R^{t}\left[1-\chi_{\tilde{L}}(R)\right] d \sigma_{R}(\zeta) d R \\
& \leq \epsilon C \int_{0}^{r_{0}} R^{t-\delta} \int_{\partial D_{R}}|K(z, \zeta)| d \sigma_{R} d R \\
& \leq \epsilon C \int_{0}^{r_{0}} \frac{R^{t-\delta}}{(r+R)^{t+1}} d R
\end{aligned}
$$




$$
\leq C \epsilon r^{-\delta}
$$

which proves estimate (13).

Due to embedding $A_{\delta}^{\infty, 1} \hookrightarrow A_{\delta}^{\infty, p}, p \geq 1$, in proving estimate (14) we can assume that $p=1$. Let us fix an $0<R<r_{0}$ and $z \in \partial D_{R}$. Using Fubini's theorem and Lemma 2 we obtain:

$$
\begin{aligned}
\left|f_{1}(z)\right| & \leq C \int_{0}^{r_{0}} \int_{\partial D_{r}}|f(\zeta)||K(z, \zeta)| d \sigma_{r}(\zeta) r^{t} \chi_{\tilde{L}}(R) d r \\
& \leq C\|f\|_{A_{\delta}^{\infty}} \int_{0}^{r_{0}} \int_{\partial D_{r}}|K(z, \zeta)| d \sigma_{r}(\zeta) r^{t-\delta} \chi_{\tilde{L}}(r) d r \\
& \leq C\|f\|_{A_{\delta}^{\infty}} \int_{0}^{r_{0}} \frac{\chi_{\tilde{L}}(r) d r}{(R+r)^{\delta+1}} .
\end{aligned}
$$

Hence we proved that

$$
\sup _{z \in \partial D_{R}}\left|f_{1}(z)\right| \leq C\|f\|_{A_{\delta}^{\infty}} \int_{0}^{r_{0}} \frac{\chi_{\tilde{L}}(r) d r}{(R+r)^{\delta+1}} .
$$

Another application of Fubini's theorem gives

$$
\begin{aligned}
\left\|f_{1}\right\|_{A_{\delta}^{\infty, 1}} & \leq C\|f\|_{A_{\delta}^{\infty}} \int_{0}^{r_{0}} \int_{0}^{r_{0}} \frac{\chi_{\tilde{L}}(r) d r}{(R+r)^{\delta+1}} R^{\delta-1} d R \\
& \leq C\|f\|_{A_{\delta}^{\infty}} \int_{0}^{r_{0}} \chi_{\tilde{L}}(r) \frac{d r}{r}
\end{aligned}
$$

and the proof is complete.

Let us add two one-sided estimates of distances, both of them follow easily from sharp results on distances from [18] and embedding results from [14], in particular Corollary 2.8. Let us set, for $\epsilon>0$ and $s>-1$ :

$$
\Omega_{\epsilon, s}=\Omega_{\epsilon, s}(f)=\left\{z \in D:|f(z)| \rho(z)^{s} \geq \epsilon\right\} .
$$

Proposition 4. Let $0<p \leq q \leq 1, s q>n, t_{0}=\frac{s+n+1}{q}$ and $f \in A_{s}^{\infty}(D)$. Set

$$
\omega_{1}=\operatorname{dist}_{A_{s}^{\infty}}\left(f, A_{s q-n}^{q, p}\right)
$$

and

$\omega_{2}=\inf \left\{\epsilon>0: \int_{D}\left(\int_{\Omega_{\epsilon, s}}|K(z, \zeta)| \rho^{t-s}(\zeta) d V(\zeta)\right)^{q} \rho(z)^{s q-n-1}(z) d V(z)<\infty\right\}$, where $K$ is a Bergman kernel of type $t, t>t_{0}$. Then $\omega_{2} \leq C \omega_{1}$.

Proposition 5. Let $q>1, p \leq q \leq \infty, s q>n$ and $t_{0}=\max \left(s, \frac{s+n+1}{q}\right)$. Set, for $f \in A_{s}^{\infty}(D)$ :

$$
w_{1}=\operatorname{dist}_{A_{s}^{\infty}}\left(f, A_{s q-n}^{q, p}\right)
$$

and

$w_{2}=\inf \left\{\epsilon>0: \int_{D}\left(\int_{\Omega_{\epsilon, s}} \mid K(z, \zeta) p^{t-s}(\zeta) d V(\zeta)\right)^{q} \rho(z)^{s q-n-1}(z) d V(z)<\infty\right\}$, 
where $K$ is a Bergman kernel of type $t, t>t_{0}$. Then $w_{2} \leq C w_{1}$.

We note that $A_{s q-n}^{q, p}=A_{s q-n+m q, m q}^{q, p}$, see [14].

\section{Atomic decompositions and embeddings in multifunctional spaces}

In this section we obtain embedding results for multifunctional spaces and, as corollaries, atomic decomposition results for such spaces. We need the following lemma which is contained in Corollary 3.5 from [3].

Lemma 4 ([3]). Let $0<p \leq 1, s>-1, r>0$ and $t=p(s+n+1)-(n+1)$. Then we have

$$
\left.\left.\left|\int_{D}\right| f(\xi)|| \tilde{\Phi}(z, \xi)\right|^{r} d(\xi)^{s} d V(\xi)\right|^{p} \leq C \int_{D}|f(\xi)|^{p}|\tilde{\Phi}(z, \xi)|^{r p} d(\xi)^{t} d V(\xi) .
$$

Clearly this lemma is also valid if $|\tilde{\Phi}|^{r}$ is replaced by $K$, where $K$ is a kernel of type $r>0$. Also, $d$ can be replaced by $\rho$ since $d(\xi) \asymp \rho(\xi)$.

Theorem 5. Let $\tilde{p}=(p, \ldots, p)$ where $0<p<\infty, \beta_{j} \in \mathbb{R}, \alpha_{j}>-1$ and $f_{j} \in H(D)$ for $1 \leq j \leq m$. Assume $\left(f_{1}, \ldots, f_{m}\right) \in M A_{\tilde{p}, \tilde{\alpha}}(D), \sum_{j=1}^{m} \beta_{j}>-1$ and the following representation holds:

$$
f_{1}\left(z_{1}\right) \cdots f_{m}\left(z_{m}\right)=C \int_{D} \rho(z)^{\frac{1}{m} \sum_{j=1}^{m} \beta_{j}} \prod_{j=1}^{m} f_{j}(z) K_{j}\left(z, z_{j}\right) d V(z),
$$

for all $z_{j} \in D, 1 \leq j \leq m$, where $K_{j}$ is a kernel of type $\frac{n+1+\beta_{j}}{m}-n-1$, $1 \leq j \leq m$, holomorphic in $z \in D$. Set $\beta=\frac{1}{m} \sum_{j=1}^{m} \beta_{j}$. We also assume that

$$
\begin{gathered}
\min _{j} \beta_{j}>m\left(n+1+\max _{j} \alpha_{j}\right) / p-(n+1) \quad \text { for } 0<p \leq 1, \\
\min _{j} \beta_{j}>m\left(n+1+\max _{j} \alpha_{j}\right) / p+(\beta+n+1) / q-(n+1) \quad \text { for } p>1,
\end{gathered}
$$

where $q$ is the exponent conjugate to $p$. Then we have the following estimate:

$$
\prod_{j=1}^{m}\left\|f_{j}\right\|_{A_{\alpha_{j}}^{p}}^{p} \leq C\left(f_{1}, \ldots, f_{m}\right)_{\tilde{p}, \tilde{\alpha}} .
$$

Proof. Let us assume $0<p \leq 1$. We apply Lemma 4 to an analytic function $f(z)=f_{1}(z) \cdots f_{m}(z)$ that appears in (15) and obtain:

$$
\prod_{k=1}^{m}\left|f_{k}\left(z_{k}\right)\right|^{p} \leq C \int_{D} \prod_{k=1}^{m}\left|f_{k}(z)\right|^{p}\left|K_{k}\left(z, z_{k}\right)\right|^{p} \rho^{\tau}(z) d V(z),
$$

where $\tau=p(\beta+n+1)-(n+1)$. This estimate and Fubini's theorem give

$$
\prod_{k=1}^{m} \int_{D}\left|f_{k}\left(z_{k}\right)\right|^{p} \rho^{\alpha_{k}}\left(z_{k}\right) d V\left(z_{k}\right)
$$




$$
\begin{aligned}
& =\int_{D} \cdots \int_{D} \prod_{k=1}^{m}\left|f_{k}\left(z_{k}\right)\right|^{p} \prod_{k=1}^{m} \rho^{\alpha_{k}}\left(z_{k}\right) d V\left(z_{1}\right) \cdots d V\left(z_{m}\right) \\
& \leq C \int_{D} \cdots \int_{D} \int_{D} \prod_{k=1}^{m}\left|f_{k}(z)\right|^{p}\left|K_{k}\left(z, z_{k}\right)\right|^{p} \rho^{\tau}(z) d V(z) \prod_{k=1}^{m} \rho^{\alpha_{k}}\left(z_{k}\right) d V\left(z_{1}\right) \cdots d V\left(z_{m}\right) \\
& =C \int_{D} \prod_{k=1}^{m}\left|f_{k}(z)\right|^{p} \rho^{\tau}(z) \int_{D} \cdots \int_{D}\left|K_{k}\left(z, z_{k}\right)\right|^{p} \prod_{k=1}^{m} \rho^{\alpha_{k}}\left(z_{k}\right) d V\left(z_{1}\right) \cdots d V\left(z_{m}\right) d V(z) .
\end{aligned}
$$

Note that $\left|K_{k}\left(z, z_{k}\right)\right|^{p}$ is a kernel of type $\frac{p\left(n+1+\beta_{k}\right)}{m}-n-1$, therefore $m$-fold application of Lemma 2 gives

$$
\prod_{k=1}^{m} \int_{D}\left|f_{k}\left(z_{k}\right)\right|^{p} \rho^{\alpha_{k}}\left(z_{k}\right) d V\left(z_{k}\right) \leq \int_{D} \prod_{k=1}^{m}\left|f_{k}(z)\right|^{p} \rho^{(m-1)(n+1)+\sum_{k=1}^{m} \alpha_{k}}(z) d V(z),
$$

which is $(16)$.

Now we consider the case $1<p<\infty$. We again have, using assumption (15) and Fubini's theorem:

$$
\begin{aligned}
& \prod_{k=1}^{m} \int_{D}\left|f_{k}\left(z_{k}\right)\right|^{p} \rho^{\alpha_{k}}\left(z_{k}\right) d V\left(z_{k}\right) \\
= & \int_{D} \cdots \int_{D} \prod_{k=1}^{m}\left|f_{k}\left(z_{k}\right)\right|^{p} \prod_{k=1}^{m} \rho^{\alpha_{k}}\left(z_{k}\right) d V\left(z_{1}\right) \cdots d V\left(z_{m}\right) \\
\leq & C \prod_{k=1}^{m} \int_{D}\left|f_{k}\left(z_{k}\right)\right|^{p} \rho^{\alpha_{k}}\left(z_{k}\right) d V\left(z_{k}\right) \\
\leq & C \int_{D} \cdots \int_{D} I^{p}\left(z_{1}, \ldots, z_{m}\right) \prod_{k=1}^{m} \rho^{\alpha_{k}}\left(z_{k}\right) d V\left(z_{1}\right) \cdots d V\left(z_{m}\right),
\end{aligned}
$$

where

$$
I\left(z_{1}, \ldots, z_{m}\right)=\int_{D} \prod_{k=1}^{m}\left|f_{k}(z)\right| \prod_{k=1}^{m}\left|K_{k}\left(z, z_{k}\right)\right| \rho^{\beta}(z) d V(z), \quad z_{1}, \ldots, z_{m} \in D .
$$

Let us set $\lambda_{k}+\mu_{k}=1,1 \leq k \leq m$, where positive numbers $\lambda_{k}$ and $\mu_{k}$ will be chosen later. Using Holder inequality we get:

$$
\begin{aligned}
I^{p}\left(z_{1}, \ldots, z_{m}\right) \leq & \left(\int_{D} \prod_{k=1}^{m}\left|K_{k}^{q \mu_{k}}\left(z, z_{k}\right)\right| \rho^{\beta}(z) d V(z)\right)^{p / q} \\
& \int_{D} \prod_{k=1}^{m}\left|f_{k}(z)\right|^{p} \prod_{k=1}^{m}\left|K_{k}^{p \lambda_{k}}\left(z, z_{k}\right)\right| \rho^{\beta}(z) d V(z) .
\end{aligned}
$$


Applying again Holder inequality for $m$ functions we obtain with help of Lemma 2 :

$$
\begin{aligned}
\int_{D} \prod_{k=1}^{m}\left|K_{k}^{q \mu_{k}}\left(z, z_{k}\right)\right| \rho^{\beta}(z) d V(z) & \leq \prod_{k=1}^{m}\left(\int_{D}\left|K_{k}^{m q \mu_{k}}\left(z, z_{k}\right)\right| \rho^{\beta}(z) d V(z)\right)^{1 / m} \\
& \leq C \prod_{k=1}^{m} \rho^{\frac{n+1+\beta}{m}-q \mu_{k} \frac{n+1+\beta_{k}}{m}}\left(z_{k}\right) .
\end{aligned}
$$

Note that conditions $n+1+\beta<q \mu_{k}\left(n+1+\beta_{k}\right), 1 \leq k \leq m$, ensure applicability of Lemma 2. Let us set

$$
\nu_{k}=\frac{p}{m}\left(\frac{n+1+\beta}{q}-\mu_{k}\left(n+1+\beta_{k}\right)\right), \quad k=1, \ldots, m .
$$

We combine the above estimates and use $m$ times Lemma 2 :

$$
\begin{aligned}
& \prod_{k=1}^{m} \int_{D}\left|f_{k}\left(z_{k}\right)\right|^{p} \rho^{\alpha_{k}}\left(z_{k}\right) d V\left(z_{k}\right) \\
\leq & C \int_{D} \cdots \int_{D} \prod_{k=1}^{m} \rho\left(z_{k}\right)^{\alpha_{k}+\nu_{k}} \int_{D} \prod_{k=1}^{m}\left|f_{k}(z)\right|^{p} \prod_{k=1}^{m}\left|K_{k}^{p \lambda_{k}}\left(z, z_{k}\right)\right| \rho^{\beta}(z) d V(z) \\
& d V\left(z_{1}\right) \cdots d V\left(z_{m}\right) \\
\leq & C \int_{D} \prod_{k=1}^{m}\left|f_{k}(z)\right|^{p} \rho(z)^{\theta} d V(z)
\end{aligned}
$$

where

$$
\begin{aligned}
\theta & =\beta+\sum_{k=1}^{m}\left(\alpha_{k}+(n+1)+\nu_{k}-p \lambda_{k} \frac{n+1+\beta_{k}}{m}\right) \\
& =\beta+\sum_{k=1}^{m}\left(\alpha_{k}+\frac{p}{m}\left(\frac{n+1+\beta}{q}-\mu_{k}\left(n+1+\beta_{k}\right)\right)-p \lambda_{k} \frac{n+1+\beta_{k}}{m}+n+1\right) \\
& =\sum_{k=1}^{m} \alpha_{k}
\end{aligned}
$$

as required. It is easily seen that all the applications of Lemma 2 can be justified by a judicious choice of $\lambda_{k}$ and $\mu_{k}$.

It is natural question to ask if an analogue of the estimate (16) is valid for general $\tilde{p}=\left(p_{1}, \ldots, p_{m}\right)$. lary.

Using Theorem 1 we immediately obtain the first part of the following corol-

Corollary 1. Assume, in addition to the assumptions of Theorem 5, that none of the functions $f_{j}$ is identically zero and assume

$$
b>\frac{n \max (p, 1)+\max \alpha_{k}+1}{p} .
$$


Let $K_{b, k}$ be the Bergman kernel of type $b$ for $A_{\alpha_{k}}^{p}$. Then there is a sequence $a_{j}$ of points in $D$ such that each of the functions $f_{k}$ admits atomic decomposition:

$$
f_{k}(z)=\sum_{j=1}^{\infty} c_{j}^{(k)} K_{b, k}\left(z, a_{j}\right), \quad k=1, \ldots, m .
$$

Moreover, $\left\|c^{(k)}\right\|_{l^{p}} \asymp\left\|f_{k}\right\|_{A_{\alpha_{k}}^{p}}$.

Conversely, if each of the functions $f_{1}, \ldots, f_{m}$ has representation as in (17), then we have:

$$
\left(f_{1}, \ldots, f_{m}\right)_{\tilde{p}, \tilde{\alpha}} \leq C \prod_{j=1}^{m}\left\|f_{j}\right\|_{A_{\alpha_{j}}^{p}}^{p} .
$$

Proof. We prove the second statement by induction on $m$. The $m=1$ case follows from [14], Theorem 4.1. Let us assume the statement is true for $m-1$ functions. Using the following well known estimate:

$$
|f(z)| \leq C\|f\|_{A_{\alpha}^{p}} d(z)^{-\frac{n+1+\alpha}{p}}, \quad z \in D,
$$

valid for $\alpha>-1$ and $0<p<\infty$ we obtain, using inductive hypothesis, the following estimate:

$$
\begin{aligned}
\left(f_{1}, \ldots, f_{m}\right)_{\tilde{p}, \tilde{\alpha}}^{p}= & \int_{D}\left|\prod_{k=1}^{m} f_{k}(z)\right|^{p} \rho(z)^{(m-1)(n+1)+\sum_{j=1}^{m} \alpha_{j}} d V(z) \\
\leq & C \sup _{z \in D}\left|f_{m}(z)\right|^{p} d(z)^{n+1+\alpha_{m}} \\
& \int_{D}\left|\prod_{k=1}^{m-1} f_{k}(z)\right|^{p} \rho(z)^{(m-2)(n+1)+\sum_{j=1}^{m-1} \alpha_{j}} d V(z) \\
\leq & C\left\|f_{m}\right\|_{A_{\alpha_{m}}^{p}}^{p} \prod_{k=1}^{m-1}\left\|f_{k}\right\|_{A_{\alpha_{k}}^{p}}^{p} .
\end{aligned}
$$

Note that the above corollary is a direct generalization of Theorem 1 to the multifunctional weighted Bergman space case.

Now we turn to atomic decomposition of multifunctional Bloch space. Lemma 6 below is, in the case $D=\mathbb{B}^{n} \subset \mathbb{C}^{n}$ contained in [11] and, for strictly pseudoconvex domains but without logarithmic factor, in [1].

Lemma 5. Let $\alpha \geq 0, t>s>0$ and $A>0$. Then

$$
\int_{0}^{A} \int_{\mathbb{R}}(r+x+|y|)^{-t-2} x^{s} \log ^{\alpha} \frac{A}{x} d y d x \leq C r^{s-t} \log ^{\alpha} \frac{A}{r}, \quad r>0 .
$$

Proof. We integrate at first with respect to $y \in \mathbb{R}$ and obtain

$$
I(A, \alpha, t, s)=\int_{0}^{A} \int_{\mathbb{R}}(r+x+|y|)^{-t-2} x^{s} \log ^{\alpha} \frac{A}{x} d y d x
$$




$$
\begin{aligned}
& \leq C \int_{0}^{A}(r+x)^{-t-1} x^{s} \log ^{\alpha} \frac{A}{x} d x \\
& =C r^{s-t} \log ^{\alpha} \frac{A}{r} \int_{0}^{A / r} \frac{u^{s}}{(1+u)^{t+1}}\left(1+\frac{\log \frac{A}{u}}{\log \frac{A}{r}}\right)^{\alpha} d u \\
& \leq C r^{s-t} \log ^{\alpha} \frac{A}{r} \int_{0}^{\infty} \frac{u^{s}}{(1+u)^{t+1}}\left(1+\log \frac{A}{u}\right)^{\alpha} d u \\
& =C r^{s-t} \log ^{\alpha} \frac{A}{r} .
\end{aligned}
$$

Lemma 6. Let $K$ be a kernel of type $t$ and let $0<s<t$. Set $D_{0}=\operatorname{diam}(D)$. Then, for every $k \in \mathbb{N}$ we have the following estimate:

$$
\int_{D}|K(z, \eta)| \mid d(\eta)^{s} \log ^{k} \frac{D_{0}}{d(\eta)} d V(\eta) \leq \frac{C}{d(z)^{t-s}} \log ^{k} \frac{D_{0}}{d(z)}, \quad z \in D .
$$

Proof. We follow the method used to prove Theorem 2.7 from [1], which is closely related to arguments from [6] and we refer the reader to these two papers for more details. First, for every $z_{0} \in \partial D$ there is a neighborhood $U$ of $z_{0}$ and a complex coordinate system $\phi=\left(\phi_{1}, \ldots, \phi_{n}\right): U \rightarrow \mathbb{C}^{n}$ such that

$$
|K(z, w)| \leq C\left(\rho(z)+\rho(w)+\left|\phi_{1}(z)-\phi_{1}(w)\right|+\sum_{k=2}^{n}\left|\phi_{k}(z)-\phi_{k}(w)\right|^{2}\right)^{-(n+1+t)}
$$

for all $z, w \in U$. Using a standard compactness argument and smoothness of the kernel $K$ in both variables inside $D$, we can localize our problem near $z_{0}$, i.e., it suffices to prove that

$$
\int_{U \cap D} \mid K(z, \eta) \| d(\eta)^{s} \log ^{k} \frac{D_{0}}{d(\eta)} d V(\eta) \leq \frac{C}{d(z)^{t-s}} \log ^{k} \frac{D_{0}}{d(z)} .
$$

Set $\tilde{U}=\phi(U \cap D)$. We use the coordinate system $\phi=\left(\phi_{1}, \ldots, \phi_{n}\right)$ in the change of variables formula and (19) to obtain

$$
\begin{aligned}
& \int_{U \cap D}|K(z, \eta)| \mid d(\eta)^{s} \log ^{k} \frac{D_{0}}{d(\eta)} d V(\eta) \\
\leq & C \int_{U \cap D}\left(\rho(z)+\rho(\eta)+\left|\phi_{1}(z)-\phi_{1}(\eta)\right|+\sum_{k=2}^{n}\left|\phi_{k}(z)-\phi_{k}(\eta)\right|^{2}\right)^{-(n+1+t)} \\
& d(\eta)^{s} \log ^{k} \frac{D_{0}}{d(\eta)} d V(\eta) \\
\leq & C \int_{\tilde{U}}\left(\rho(z)+\tilde{\rho}(\eta)+\left|z_{1}-\eta_{1}\right|+\sum_{k=2}^{n}\left|z_{k}-\eta_{k}\right|^{2}\right)^{-n-1-t} d(\eta)^{s} \log ^{k} \frac{D_{0}}{d(\eta)} d V(\eta),
\end{aligned}
$$


where $\tilde{\rho}=\rho \circ \phi^{-1}$. Using another change of variables, see [1], one estimates the last integral by a constant multiple of

$$
I=\int_{W}\left(\rho(z)+x+|y|+\sum_{k=2}^{n}\left|w_{k}\right|^{2}\right)^{-(n+1+t)} x^{s} \log ^{k} \frac{D_{0}}{x} d w_{2} \cdots d w_{n} d x d y
$$

where $W=\left[0, d_{0}\right] \times \mathbb{R} \times \mathbb{C}^{n-1}$. Using arguments from [1] and the previous lemma one arrives at the desired estimate (18).

Theorem 6. Let $f_{k} \in H(D), 1 \leq k \leq m$, assume none of the functions $f_{j}$ is identically zero in $D$ and $\left(f_{1}, \ldots, f_{m}\right)_{M B(m)}<\infty$.

a) Assume there are constants $\beta_{i, j}>0$ and kernels $K_{i, j}(\zeta, z)$ of type $\frac{n+1+\beta_{i, j}}{m}$ $-n-1,1 \leq i, j \leq m$ such that for all $i=1, \ldots, m$ we have an integral representation:

$$
\begin{aligned}
& \nabla f_{i}\left(z_{i}\right) \prod_{j \neq i} f_{j}\left(z_{j}\right) \\
= & C_{i}\left(\int_{D} \nabla\left(f_{1} \ldots f_{m}\right)(z) \rho(z)^{\frac{1}{m} \sum_{j=1}^{m} \beta_{i, j}} \prod_{j=1}^{m} K_{i j}\left(z_{j}, z\right) d V(z)\right)^{m},
\end{aligned}
$$

where $z_{j} \in D$ for $j=1, \ldots, m$. Then we have $f_{j} \in \mathcal{B}(D)$ for each $1 \leq j \leq m$.

b) Assume there are constants $\beta_{i, j}>0$ and kernels $K_{i, j}(\zeta, z)$ of type $\frac{n+m+\beta_{i, j}}{m}$ $-n-1,1 \leq i, j \leq m$ such that for all $i=1, \ldots, m$ we have an integral representation:

$$
\nabla f_{i}\left(z_{i}\right) \prod_{j \neq i} f_{j}\left(z_{j}\right)=C_{i} \int_{D} \nabla\left(f_{1} \ldots f_{m}\right)(z) \rho(z)^{\frac{1}{m} \sum_{j=1}^{m} \beta_{i, j}} \prod_{j=1}^{m} K_{i j}\left(z_{j}, z\right) d V(z)
$$

where $z_{j} \in D$ for $j=1, \ldots, m$. Then we have $f_{j} \in \mathcal{B}(D)$ for each $1 \leq j \leq m$.

We note that conditions (21) and (22) coincide for $m=1$ and are satisfied for any $f \in \mathcal{B}$. Indeed, if $f \in \mathcal{B}(D)$, then $\nabla f \in A_{1}^{\infty}(D) \hookrightarrow A_{1}^{1}(D)$ and we can use the Bergman representation formula for $\nabla f$.

Proof. Let us prove that $f_{1} \in \mathcal{B}$, under assumptions in a). Using (21) we immediately obtain:

$$
\begin{aligned}
& \left|\nabla f_{1}\left(z_{1}\right)\right| \prod_{j=2}^{n}\left|f_{j}\left(z_{j}\right)\right| \\
\leq & C\left(f_{1}, \ldots, f_{m}\right)_{M B}^{m}\left(\int_{D} \log ^{m-1} \frac{D_{0}}{d(z)} d(z)^{-1+\sum_{j=1}^{m} \beta_{1, j}} \prod_{j=1}^{m}\left|K_{1, j}\left(z_{j}, z\right)\right| d V(z)\right)^{m} .
\end{aligned}
$$


Using Hölder inequality for $m$ functions and the previous lemma we obtain:

$$
\begin{aligned}
\left|\nabla f_{1}\left(z_{1}\right) \prod_{j=2}^{m} f_{j}\left(z_{j}\right)\right|^{1 / m} & \leq C\left(\int_{D}\left|K_{1,1}\left(z, z_{1}\right)\right|^{m} d(z)^{\beta_{1,1}-1} d V(z)\right)^{1 / m} \\
& \prod_{j=2}^{m}\left(\int_{D} \log ^{m} \frac{D_{0}}{d(z)} d(z)^{\beta_{1, j}-1}\left|K_{1, j}\left(z, z_{j}\right)\right|^{m} d V(z)\right)^{1 / m} \\
& \leq C d\left(z_{1}\right)^{-1 / m} \prod_{j=2}^{m} d\left(z_{j}\right)^{-1 / m} \log \frac{D_{0}}{d\left(z_{j}\right)}
\end{aligned}
$$

and this clearly gives $\left|\nabla f_{1}(w)\right| \leq C d(w)^{-1}$, and therefore $f_{1} \in \mathcal{B}$. Similarly one proves $f_{j} \in \mathcal{B}$ for all $j \geq 2$.

Next, let us prove $f_{1} \in \mathcal{B}$ assuming (22). Now we have, using Fubini's theorem:

$$
\begin{aligned}
& \left|\nabla f_{1}\left(z_{1}\right)\right| \prod_{j=2}^{n}\left|f_{j}\left(z_{j}\right)\right| \\
\leq & C\left(f_{1}, \ldots, f_{m}\right)_{M B} \int_{D} \log ^{m-1} \frac{D_{0}}{d(z)} d(z)^{-1+\sum_{j=1}^{m} \beta_{1, j}} \prod_{j=1}^{m}\left|K_{1, j}\left(z_{j}, z\right)\right| d V(z) \\
\leq & C\left(\int_{D}\left|K_{1,1}\left(z, z_{1}\right)\right|^{m} d(z)^{\beta_{1,1}-1} d V(z)\right)^{1 / m} \\
& \prod_{j=2}^{m}\left(\int_{D} \log ^{m} \frac{D_{0}}{d(z)} d(z)^{\beta_{1, j}-1}\left|K_{1, j}\left(z, z_{j}\right)\right|^{m} d V(z)\right)^{1 / m} \\
\leq & C d\left(z_{1}\right) \prod_{j=2}^{m} d\left(z_{j}\right) \log \frac{D_{0}}{d\left(z_{j}\right)},
\end{aligned}
$$

and this, as in part a), suffices.

Atomic decomposition of $\mathcal{B}(D)$ was obtained in [5]. The first part of the following corollary generalizes this result to the multifunctional Bloch space on $D$ and, moreover, follows immediately from it, in view of the above embedding theorem.

Corollary 2. Assume, in addition to the assumptions of the above theorem, that $b>n$ and that $\left\{a_{l, j}\right\}$ is an $\eta_{0}-\eta_{1}$ lattice in $D$. Then for every $k=1, \ldots, m$ there is a bounded double sequence $\left\{c_{l, j}^{k}\right\}$ such that

$$
f_{k}(z)=\sum_{l, j} c_{l, j}^{k} \frac{d\left(a_{l, j}^{k}\right)^{b}}{K\left(a_{l, j}^{k}, z\right)}, \quad z \in D, \quad 1 \leq k \leq m
$$


where $K$ is a kernel of type $b-n-1$. Conversely, if $f_{k} \in H(D), 1 \leq k \leq m$, and every $f_{k}$ admits representation as in (23), with a bounded double sequence $\left\{c_{l, j}^{k}\right\}$, then $\left(f_{1}, \ldots, f_{m}\right)_{M B(m)}<\infty$.

Proof. Let us prove the second statement. We have $f_{k} \in \mathcal{B}$ for all $k=1, \ldots, m$ and therefore we have the following growth estimate:

$$
\left|f_{k}(z)\right| \leq C\left\|f_{k}\right\|_{\mathcal{B}} \log d(z)^{-1}
$$

for $0<d(z)<1$. Hence

$$
\begin{aligned}
\left(f_{1}, \ldots, f_{m}\right)_{M B(m)} & =\sup _{z \in D}\left|\nabla\left(f_{1} \ldots f_{m}\right)(z)\right| d(z) \log ^{1-m} \frac{1}{d(z)} \\
& \leq C \sup _{z \in D} \sum_{k=1}^{m}\left|\nabla f_{k}(z)\right| \prod_{j \neq k}\left|f_{j}(z)\right| \log ^{1-m} \frac{1}{d(z)} \\
& \leq C \prod_{k=1}^{m}\left\|f_{k}\right\|_{\mathcal{B}} .
\end{aligned}
$$

\section{References}

[1] M. Abate, J. Raissy, and A. Saracco, Toeplitz operators and Carleson measures in strongly pseudoconvex domains, J. Funct. Anal. 263 (2012), no. 11, 3449-3491.

[2] P. Ahern and R. Schneider, Holomorphic Lipschitz functions in pseudoconvex domains, Amer. J. Math. 101 (1979), no. 3, 543-565.

[3] F. Beatrous, Jr. $L^{p}$-estimates for extensions of holomorphic functions, Michigan Math. J. 32 (1985), no. 3, 361-380.

[4] W. S. Cohn, Weighted Bergman projections and tangential area integrals, Studia Math. 106 (1993), no. 1, 59-76.

[5] R. Coifman and R. Rochberg, Representation theorems for holomorphic and harmonic functions in $L^{p}$, Representation theorems for Hardy spaces, pp. 11-66, Astérisque, 77, Soc. Math. France, Paris, 1980.

[6] Ž. Čučković and J. D. McNeal, Special Toeplitz operators on strongly pseudoconvex domains, Rev. Mat. Iberoam. 22 (2006), no. 3, 851-866.

[7] P. Duren, Theory of $H^{p}$ Spaces, Academic Press, 1970.

[8] N. Kerzman and E. M. Stein, The Szegö kernel in terms of Cauchy-Fantappie kernels, Duke Math. J. 45 (1978), no. 2, 197-223.

[9] S. Li and W. Luo, On characterization of Besov space and application. Part I, J. Math. Anal. Appl. 310 (2005), 477-491.

[10] _ Analysis on Besov spaces. II. Embedding and duality theorems, J. Math. Anal. Appl. 333 (2007), no. 2, 1189-1202.

[11] S. Li and R. Shamoyan, On some extensions of theorems on atomic decompositions of Bergman and Bloch spaces in the unit ball and related problems, Complex Var. Elliptic Equ. 54 (2009), no. 12, 1151-1162.

[12] _ On some estimates and Carleson type measure for multifunctional holomorphic spaces in the unit ball, Bull. Sci. Math. 134 (2010), no. 2, 144-154.

[13] E. Ligocka, On the Forelli-Rudin construction and weighted Bergman projections, Studia Math. 94 (1989), no. 3, 257-272.

[14] J. M. Ortega and J. Fabrega, Mixed-norm spaces and interpolation, Studia Math. 109 (1994), no. 3, 233-254 
[15] M. M. Peloso, Hankel operators on weighted Bergman spaces on strongly pseudoconvex domains, Illinois J. Math. 38 (1994), no. 2, 223-249.

[16] R. M. Range, Holomorphic Functions and Integral Representations in Several Complex Variables, Graduate Texts in Mathematics, 108. Springer-Verlag, New York, 1986.

[17] R. F. Shamoyan, Some remarks on the action of Lusin area operator in Bergman spaces of the unit ball, Acta Univ. Apulensis Math. Inform. 29 (2012), 31-45.

[18] R. F. Shamoyan and M. Arsenović, Some remarks on extremal problems in weighted Bergman spaces of analytic functions, Commun. Korean Math. Soc. 27 (2012), no. 4, $753-762$,

[19] R. Shamoyan and O. Mihić, On new estimates for distances in analytic function spaces in higher dimension, Sib. Ėlektron. Mat. Izv. 6 (2009), 514-517.

[20] _ On new estimates for distances in analytic function spaces in the unit disk, the polydisk and the unit ball, Bol. Asoc. Mat. Venez. 17 (2010), no. 2, 89-103.

[21] K. Zhu, Spaces of Holomorphic Functions in the Unit Ball, Springer-Verlag, New York, 2005.

Miloš Arsenović

BRYANSK UNIVERSITY

BRYANSK, RUSSIA

E-mail address: rshamoyan@yahoo.com

ROMI F. SHAMOYAN

FACULTY OF MATHEMATICS

UNIVERSITY OF BELGRADE

Studentski Trg 16, 11000 Belgrade, Serbia

E-mail address: arsenovic@matf.bg.ac.rs 